\title{
Avaliação do perfil lipídico em pacientes com doença inflamatória intestinal
}

\author{
Evaluation of lipid profile in patients with inflammatory bowel disease
}

\author{
Elaine de Fatima Adorne ${ }^{1} \bowtie$, Luiz Carlos Bodanese ${ }^{1}$ \\ ${ }^{1}$ Programa de Pós-Graduação em Medicina e Ciências da Saúde da Faculdade de Medicina da Pontifícia Universidade Católica do Rio Grande do Sul. \\ Porto Alegre, RS.
}

\section{RESUMO}

Objetivos: Avaliar o perfil lipídico e parâmetros inflamatórios de pacientes com doença de Crohn e retocolite ulcerativa, e correlacionar com os níveis de atividade das doenças.

Métodos: Estudo transversal com pacientes acompanhados no Ambulatório de Doença Inflamatória Intestinal do Hospital São Lucas da PUCRS. Foram avaliadas as características demográficas, antropométricas e metabólicas dos pacientes com doença inflamatória intestinal crônica. Avaliaram-se parâmetros lipídios e inflamatórios e analisou-se a correlação entre a gravidade da doença inflamatória e os parâmetros metabólicos, na doença de Crohn pelo índice de Harvey-Bradshaw e na retocolite ulcerativa pelo método de Truelove e Witts.

Resultados: Participaram do estudo 122 pacientes de ambos os sexos com doença inflamatória intestinal, estratificados em: doença de Crohn $(n=64)$ e retocolite ulcerativa $(n=58)$. As mulheres representaram $54,1 \%$ da amostra, a média de idade foi de $41,6 \pm 12,6$ anos e a média de índice de massa corporal foi de $25 \pm 4,4 \mathrm{~kg} / \mathrm{m}^{2}$. O colesterol ligado à lipoproteína de baixa densidade (LDL-c) mostrou-se significativamente mais elevado nos pacientes com retocolite ulcerativa comparando-se aos pacientes com doença de Crohn $(\mathrm{p}=0,006)$. A proteína $\mathrm{C}$ reativa aumentada esteve associada com maior gravidade do processo inflamatório em pacientes com doença de Crohn ( $\mathrm{p}=0,027)$, quando analisada pelo índice de Harvey-Bradshaw. Na retocolite ulcerativa, conforme o índice de Truelove e Witts, houve correlação fraca entre atividade da doença e colesterol ligado à lipoproteína de alta densidade (HDL-c) $\left(\mathrm{r}_{\mathrm{s}}=-0,29 ; \mathrm{p}=0,036\right)$ e entre atividade da doença e colesterol total $\left(r_{s}=-0,30 ; p=0,028\right)$. Cerca de dois terços dos pacientes $(67,6 \%)$ apresentaram o HDL-c abaixo dos valores recomendados.

Conclusões: O LDL-c mostrou-se significativamente mais elevado nos pacientes com retocolite ulcerativa comparados aos pacientes com doença de Crohn. O HDL-c apresentou níveis reduzidos em pacientes com doença inflamatória mais grave na retocolite ulcerativa. A proteína $\mathrm{C}$ reativa aumentada em pacientes com doença de Crohn esteve associada com a gravidade do processo inflamatório determinada pelo índice de Harvey-Bradshaw. A detecção dessas alterações metabólicas pode auxiliar na identificação de indivíduos com potencial risco para eventos cardiovasculares

DESCRITORES: doença de Crohn; retocolite ulcerativa; dislipidemias.

\section{ABSTRACT}

Aims: To evaluate the lipid profile and inflammatory parameters in patients with Crohn's disease (CD), and ulcerative colitis (UC) and correlate them with disease activity levels.

Methods: Cross-sectional study of patients followed up at the Inflammatory Bowel Disease Outpatient Clinic of São Lucas Hospital at Pontifícia Universidade Católica do Rio Grande do Sul, Brazil. The demographic, anthropometric, and metabolic characteristics of patients with chronic inflammatory disease were evaluated. Lipids and inflammatory parameters were evaluated as well as the correlation between the severity of inflammatory disease and metabolic parameters using the Harvey-Bradshaw index for CD and Truelove and Witts' method for UC. Results: The study included 122 male and female patients with inflammatory bowel disease classified into CD ( $\mathrm{n}=64)$ and UC ( $\mathrm{n}=58$ ). Women accounted for $54.1 \%$ of the sample, with a mean age of $41.6 \pm 12.6$ years and a BMI of $25 \pm 4.4 \mathrm{~kg} / \mathrm{m}^{2}$. Low-density lipoprotein cholesterol (LDL-c) was significantly higher in UC than in $\mathrm{CD}(\mathrm{p}=0.006)$. Higher $\mathrm{C}$-reactive protein levels were associated with a more severe inflammatory process in CD patients ( $\mathrm{p}=0.027$ ) according to the Harvey-Bradshaw index. In UC, there was a weak correlation between disease activity and high-density lipoprotein cholesterol (HDL-c) $\left(r_{s}=-0.29 ; p=0.036\right)$ and between disease activity and total cholesterol $\left(r_{s}=-0.30 ; p=0.028\right)$ according to Truelove and Witts' index. The HDL-c of about two thirds of the patients $(67.6 \%)$ was below the recommended values.

Conclusions: LDL-c was significantly higher in patients with UC than in those with CD. HDL-c was lower in patients with more severe inflammatory disease triggered by UC. Increased C-reactive protein levels in CD patients were associated with severity of the inflammatory process determined by the Harvey-Bradshaw index. The detection of these metabolic changes may help identify individuals with potential risk for cardiovascular events.

KEY WORDS: Crohn disease; rectocolitis, ulcerative; dyslipidemias. 
Abreviaturas: DII, doenças inflamatórias intestinais; DC, doença de Crohn; RCU, retocolite ulcerativa; IMC, índice de massa corporal; IHB, índice de atividade inflamatória Harvey e Bradshaw; VHS, velocidade de hemossedimentação; PCR, proteína $\mathrm{C}$ reativa; $\mathrm{TG}$, triglicerídeos; LDL-c, colesterol ligado à lipoproteína de baixa densidade; HDL-c, colesterol ligado à lipoproteína de alta densidade; CT, colesterol total; DAC, doença arterial coronariana; $\mathrm{TNF} \alpha$, fator de necrose tumoral alfa; IL-6, interleucina-6.

\section{INTRODUÇÃO}

Doenças inflamatórias intestinais (DII) são doenças crônicas que cursam com períodos de exacerbação e remissão, comprometendo o sistema gastrointestinal, sendo que as mais frequentes são a doença de Crohn (DC) e a retocolite ulcerativa (RCU). Enquanto a RCU, mais localizada, compromete cólon e reto, a DC ocorre em qualquer parte do trato gastrointestinal, sendo uma doença mais difusa, podendo acometer todo o sistema digestivo [1].

ADC apresenta-se em qualquer faixa etária, com predominância em adulto jovem (dos 20 aos 40 anos) [2]. Além dos parâmetros clínicos, alguns parâmetros laboratoriais, como hemograma, velocidade de hemossedimentação (VHS) e proteína C reativa (PCR), são úteis para o diagnóstico, identificação de doença ativa e reconhecimento do risco de recaída [3-5]. Para avaliação clínica, em geral é utilizado o índice de atividade inflamatória de Harvey-Bradshaw (IHB) [6].

A RCU apresenta dois picos de incidência, sendo o primeiro entre os 15 e 30 anos e o segundo entre 50 e 70 anos. Níveis elevados da taxa de VHS e da PCR guardam relação com a intensidade do fenômeno inflamatório. O índice de Truelove e Witts é um importante recurso para avaliar e definir a gravidade do processo inflamatório dessa doença $[7,8]$.

As dislipidemias são alterações metabólicas dos lipídeos que ocasionam modificações nos níveis séricos das lipoproteínas: elevação do colesterol total (CT), do colesterol ligado a lipoproteínas de baixa densidade (LDL-c) e dos triglicerídeos (TG), e decréscimo do colesterol ligado a lipoproteínas de alta densidade (HDL-c). Existem robustas evidências de que alterações nas concentrações séricas de colesterol influenciam na ocorrência de doença arterial coronariana (DAC) [9].

Os estudos são inconclusivos em relação ao risco de eventos cardiovasculares em pacientes com DII. Porém, tanto nas DII como na DAC, os marcadores inflamatórios como interleucina-6 (IL-6), fator de necrose tumoral alfa (TNF- $\alpha$ ) e PCR apresentam-se elevados, sugerindo um mecanismo inflamatório em comum [10]. Um estudo com 67 pacientes portadores de DII, comparados a um grupo controle, evidenciou que os pacientes com DC e RCU diferenciavam-se em termos de metabolismo lipídico, e o LDL-c era menor na DC. Já no grupo controle, os pacientes apresentavam níveis reduzidos de HDL-c e o LDL-c também apresentou valores menores, sugerindo que a alteração no metabolismo lipídico pode estar relacionada à presença de inflamação crônica, mesmo com a doença em remissão [11]. A inflamação sistêmica crônica, presente em doenças como lúpus eritematoso sistêmico e artrite reumatóide, é um marcador de risco para eventos cardiovasculares. Nessas doenças, marcadores inflamatórios como PCR, TNF- $\alpha$, IL-6 e receptor CD40 estão associados a alterações nas concentrações de lipídeos, em que se verifica redução do HDL-c, hiperhomocisteinemia, resistência insulínica e disfunção endotelial [12]. Portanto, pode haver risco aumentado de eventos aterotrombóticos em pacientes com DII, já que a inflamação é uma alteração frequente na patogênese dessas doenças.

Os estudos disponíveis até o presente não permitem estabelecer com segurança associação entre alterações no metabolismo lipídico e DII. Como a inflamação está presente em pacientes com dislipidemias, que produzem alterações inflamatórias semelhantes às verificadas nas DII, a associação de ambas pode potencialmente predispor ao aumento no risco de doença aterotrombótica, especialmente DAC. Portanto, o objetivo deste estudo foi verificar a correlação entre o perfil lipídico de pacientes com DII e os níveis de atividade inflamatória da doença. Procurou-se avaliar também se há diferença no perfil lipídico entre os pacientes com DC e RCU.

\section{MÉTODOS}

Estudo transversal, cuja amostra foi composta por pacientes de ambos os sexos, com idade igual ou superior a 19 anos, portadores de DC e RCU, em condições físicas para realização de avaliação nutricional e coleta de exames laboratoriais. Os pacientes apresentavam DII em diferentes estágios de atividade inflamatória e estavam em acompanhamento no Ambulatório de Doença Inflamatória Intestinal do Hospital São Lucas da PUCRS, no período de outubro de 2014 a novembro de 2015 . Foram consideradas inelegíveis as pacientes gestantes e no puerpério.

Foram verificados dados sociodemográficos, como sexo e idade, e antropométricos, como peso e índice de 
Quadro 1. Índice de atividade inflamatória Harvey-Bradshaw na doença de Crohn, 1980.

\begin{tabular}{lc}
\hline \multicolumn{1}{c}{ Quadro clínico } & Pontuação \\
\hline Estado geral (ótimo=0; bom=1; regular=2; mau=3; péssimo=4 & $0-4$ \\
\hline Dor abdominal (ausente=0; duvidosa=1; moderada=2; grave=3 & $0-3$ \\
Número de evacuações líquidas/dia & n $/$ dia \\
Massa abdominal (ausente=0; duvidosa=1; bem definida=2; bem definida e dolorosa $=3$ & 0 a 3 \\
$\begin{array}{l}\text { Complicações: artralgia/artrite, uveíte/irite, eritema nodoso, aftas orais, pioderma gangrenoso, fissura anal, } \\
\text { fístula, abscesso, etc. }\end{array}$ & 1 ponto cada \\
\hline IHB: $<\mathbf{8}$ inativa/leve; $\mathbf{8}$ a $\mathbf{1 0}$ leve/moderada; $>\mathbf{1 0}$ moderada/grave & \\
\hline
\end{tabular}

IHB: Índice de atividade inflamatória Harvey e Bradshaw.

Fonte: Cardozo WS e Sobrado CW, 2015 [13].

Quadro 2. Classificação da gravidade do surto agudo na retocolite ulcerativa pelo índice de Truelove e Witts.

\begin{tabular}{lccc}
\multicolumn{1}{c}{ Dados clínicos } & Leve & Moderado & Grave \\
Número de evacuações/dia & $\leq 4$ & 4 a 6 & $>6$ \\
Sangue vivo nas fezes & Ausente & Presente em pequena quantidade & Presente em grande quantidade \\
Temperatura & Normal & Valores intermediários & Temperatura média noturna \\
Pulso & Normal & Valores intermediários & $>37,5^{\circ}$ ou $>37,8^{\circ}$ em 2 de 4 dias \\
Hemoglobina $(\mathrm{g} / \mathrm{dL})$ & $>10,5$ & Valores intermediários & $>90$ bpm \\
VHS (mm/1ahora) & $<30$ & Valores intermediários & $>10,5$ \\
\hline
\end{tabular}

VHS: Velocidade de hemossedimentação.

Fonte: Cardozo WS e Sobrado CW, 2015 [13].

massa corporal (IMC). Para análise do nível de atividade inflamatória da DC e da RCU, os níveis séricos de PCR e VHS foram verificados respectivamente pelos métodos de química seca e de fotometria capilar, com os seguintes valores de referência para normalidade: PCR inferior a 1,0 mg/dl; VHS até $13 \mathrm{~mm}$ para homens e até $20 \mathrm{~mm}$ para mulheres. Os pacientes foram classificados quanto à atividade inflamatória na DC por meio do IHB (Quadro 1) e quanto à gravidade do surto agudo na RCU pelo índice de Truelove e Witts (Quadro 2) [13].

Para análise do perfil lipídico foram utilizados os níveis séricos de HDL-c, LDL-c, CT e TG, verificados pelo método de química seca e fórmula de Friedewald para LDL-c, de acordo com os valores de referência para normalidade do Laboratório de Patologia Clínica do Hospital São Lucas da PUCRS, com base na recomendação da diretriz da Sociedade Brasileira de Cardiologia [9]. Os pacientes não faziam uso de medicação hipolipemiante no período do estudo.

Para análise estatística foram usados os seguintes procedimentos: distribuição binomial para categorias, média e desvio padrão para dados contínuos, análise de variância (ANOVA) para comparação de dados contínuos e coeficiente de Spearman para dados não paramétricos, utilizando o programa IBM SPSS Statistics versão 22.0, aceitando um nível de significância de $5 \%$.

O projeto foi aprovado pelo Comitê de Ética em Pesquisa da Pontifícia Universidade Católica do Rio Grande do Sul sob número 34428314600005336 em 02/10/2014. Foram utilizados somente os dados dos pacientes que aceitaram participar da pesquisa e assinaram o termo de consentimento livre esclarecido.

\section{RESULTADOS}

Participaram do estudo 122 pacientes que atenderam aos critérios de inclusão, sendo 64 pacientes $(52,5 \%)$ com DC e 58 pacientes $(47,5 \%)$ com e RCU. A média de idade foi de $41,6 \pm 12,6$ anos. As mulheres representaram $54,1 \%$ da amostra. Na Tabela 1 estão apresentadas as características demográficas, antropométricas e metabólicas da amostra. Em relação ao perfil lipídico, verificou-se que o LDL-c encontrava-se em níveis significativamente mais elevados na RCU $(110,0 \pm 35,8 \mathrm{mg} / \mathrm{dL})$ em comparação aos níveis na $\operatorname{DC}(91,3 \pm 33,1 \mathrm{mg} / \mathrm{dL})(\mathrm{p}=0,006)$. Em relação aos demais parâmetros não houve diferença (Tabela1). 
$\mathrm{Na}$ análise dos pacientes com DC, considerando-se o IHB, 37 pacientes $(63,8 \%)$ apresentavam-se com atividade inflamatória leve da doença, 15 pacientes com atividade moderada $(25,9 \%)$ e seis pacientes com atividade grave $(10,3 \%)$. A PCR esteve significativamente mais elevada $(p=0,027)$ nos pacientes com DC e atividade inflamatória grave (Tabela 2). Na DC não houve correlação significativa entre o IHB e os marcadores lipídicos: HDL-c: $r_{s}=0,001 \quad(p=0,996) ;$ LDL-c: $r_{s}=-0,16 \quad(p=0,248) ; e$ TG: $r_{s}=-0,07(p=0,565)($ Figuras 1, 2 e 3). Houve uma correlação fraca com CT: $r_{s}=-0,59(p=0,668)$ (Figura 4).
Tabela 1. Características demográficas, antropométricas e metabólicas da amostra de pacientes em atendimento no Ambulatório de Doença

Inflamatória Intestinal do Hospital São Lucas da PUCRS. Porto Alegre, outubro de 2014 a novembro de 2015.

Tabela 2. Características metabólicas dos pacientes com Doença de Crohn segundo o Índice de Harvey-Bradshaw.

\begin{tabular}{|c|c|c|c|c|}
\hline Variáveis & $\begin{array}{c}\text { DII } \\
\text { Média } \pm \text { DP } \\
(n=122)\end{array}$ & $\begin{array}{c}\text { DC } \\
\text { Média } \pm \text { DP } \\
(n=64)\end{array}$ & $\begin{array}{c}\text { RCU } \\
\text { Média } \pm \text { DP } \\
(n=58)\end{array}$ & $\mathbf{p}$ \\
\hline Feminino (n \%) & $66(54,1)$ & $39(60,9)$ & $27(46,6)$ & \\
\hline Idade (anos) & $41,6 \pm 12,6$ & $41,6 \pm 13,3$ & $41,6 \pm 12,0$ & 0,987 \\
\hline Peso (kg) & $70,1 \pm 13,5$ & $68,9 \pm 14,2$ & $71,5 \pm 12,6$ & 0,283 \\
\hline Altura (m) & $1,67 \pm 0,11$ & $1,66 \pm 0,12$ & $1,68 \pm 0,10$ & 0,148 \\
\hline IMC (kg/m²) & $25,0 \pm 4,4$ & $24,9 \pm 4,6$ & $25,0 \pm 4,1$ & 0,971 \\
\hline PCR (mg/dL) & $3,0 \pm 11,1$ & $4,1 \pm 14,9$ & $1,93 \pm 4,7$ & 0,295 \\
\hline VHS (mm) & $20,0 \pm 21,6$ & $20,1 \pm 22,9$ & $20,0 \pm 20,4$ & 0,966 \\
\hline Hemograma (g/dL) & $13,6 \pm 31,6$ & $13,4 \pm 1,4$ & $13,8 \pm 1,7$ & 0,241 \\
\hline Hematócrito (\%) & $40,3 \pm 3,8$ & $39,9 \pm 3,6$ & $40,8 \pm 3,9$ & 0,210 \\
\hline HDL-c (mg/dL) & $54,9 \pm 15,1$ & $53,6 \pm 13,9$ & $56,2 \pm 16,3$ & 0,376 \\
\hline LDL-C (mg/dL) & $100,5 \pm 35,6$ & $91,3 \pm 33,1$ & $110,0 \pm 35,8$ & 0,006 \\
\hline CT (mg/dL) & $176,9 \pm 41,8$ & $169,7 \pm 39,9$ & $184,3 \pm 42,7$ & 0,067 \\
\hline TG (mg/dL) & $120,5 \pm 72,8$ & $129,2 \pm 87,5$ & $111,3 \pm 52,3$ & 0,201 \\
\hline
\end{tabular}

DII, doença inflamatória intestinal; DC, Doença de Crohn; RCU, retocolite ulcerativa; IMC, índice de massa corporal PCR, proteína C reativa; VHS, velocidade de hemossedimentação; HDL-c, colesterol ligado à lipoproteína de alta densidade; LDL-c: colesterol ligado à lipoproteína de baixa densidade; CT: colesterol total; TG: triglicerídeos.

\begin{tabular}{lcccc}
\hline \multicolumn{1}{c}{ Variáveis } & $\begin{array}{c}\text { Leve } \\
\text { Média } \pm \text { DP } \\
(\mathbf{n}=\mathbf{3 7})\end{array}$ & $\begin{array}{c}\text { Moderada } \\
\text { Média } \pm \mathbf{D P} \\
(\mathbf{n}=\mathbf{1 5})\end{array}$ & $\begin{array}{c}\text { Grave } \\
\text { Média } \pm \mathbf{D P} \\
(\mathbf{n}=\mathbf{6})\end{array}$ & $\mathbf{p}$ \\
\hline PCR $(\mathrm{mg} / \mathrm{dL}$ & $2,1 \pm 5,29$ & $2,9 \pm 2,3$ & $19,3 \pm 43,9$ & 0,027 \\
VHS $(\mathrm{mm})$ & $16,2 \pm 15,6$ & $26,3 \pm 35,4$ & $28 \pm 18,4$ & 0,248 \\
\hline HDL-c $(\mathrm{mg} / \mathrm{dL})$ & $53,3 \pm 12,2$ & $52,9 \pm 18,2$ & $57,6 \pm 12,6$ & 0,763 \\
\hline LDL-c $(\mathrm{mg} / \mathrm{dL})$ & $96,0 \pm 34,7$ & $80,8 \pm 25,3$ & $88,8 \pm 39,1$ & 0,352 \\
CT $(\mathrm{mg} / \mathrm{dL})$ & $172,7 \pm 42,2$ & $159,5 \pm 37,4$ & $177,8 \pm 33,1$ & 0,499 \\
TG $(\mathrm{mg} / \mathrm{dL})$ & $128,5 \pm 80,1$ & $145,6 \pm 110,3$ & $92,3 \pm 63,8$ & 0,459 \\
\hline
\end{tabular}

PCR, proteína C-reativa; VHS, velocidade de hemossedimentação; HDL-c, colesterol ligado à lipoproteína de alta densidade; LDL-c, colesterol ligado à lipoproteína de baixa densidade; CT, colesterol total; TG, triglicerídeos.

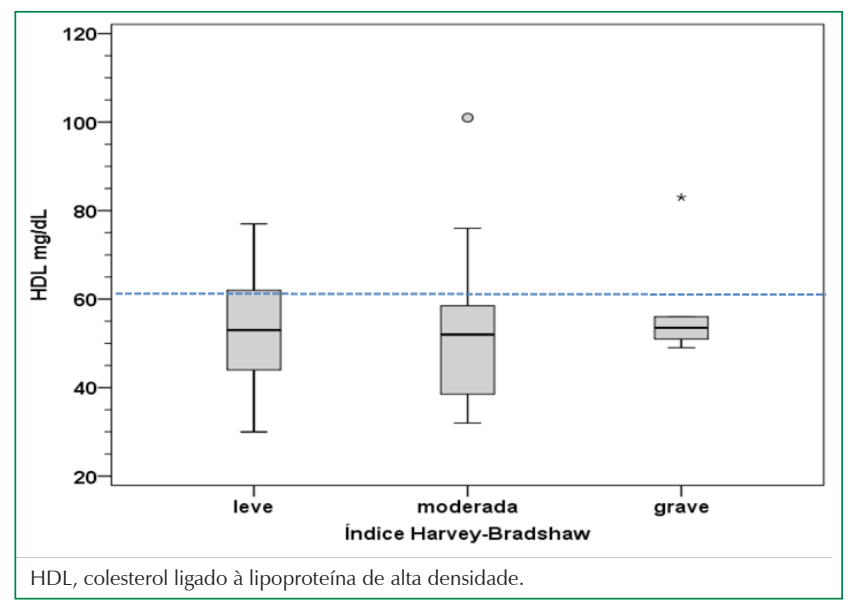

Figura 1. Comparação entre as médias de colesterol ligado à lipoproteína de alta densidade de acordo com a classificação da atividade inflamatória da doença de Crohn pelo Índice de Harvey-Bradshaw.

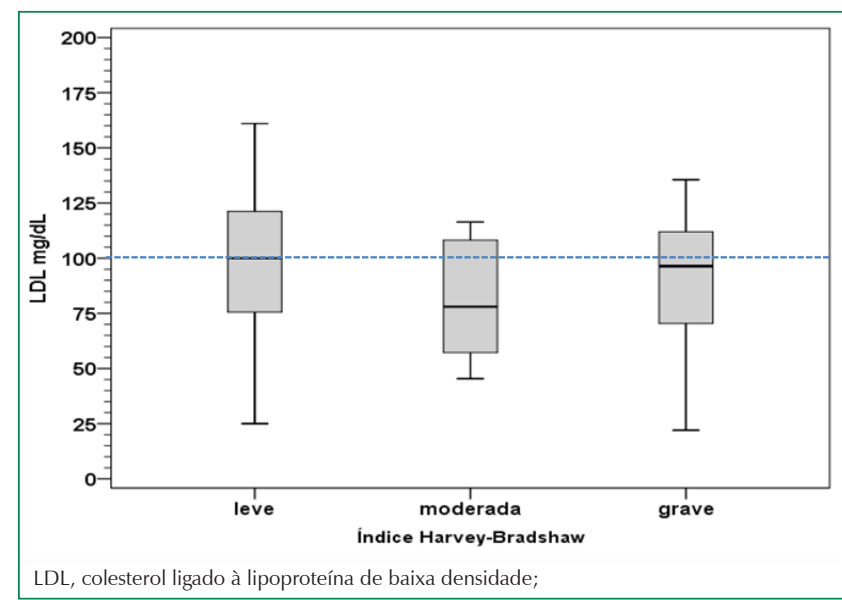

Figura 2. Comparação entre as médias de colesterol ligado à lipoproteína de baixa densidade de acordo com a classificação da atividade inflamatória da doença de Crohn pelo Índice de Harvey-Bradshaw. 


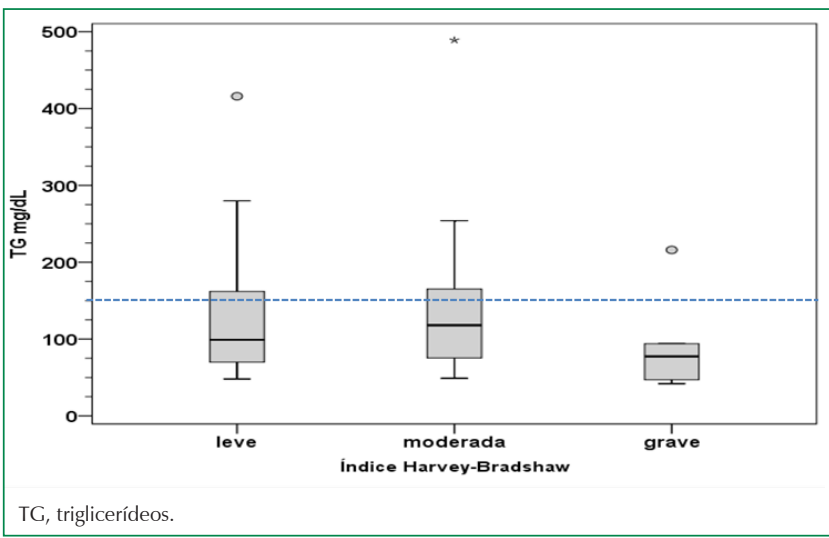

Figura 3. Comparação entre as médias dos triglicerídeos de acordo com a classificação da atividade inflamatória da doença de Crohn pelo Índice de Harvey-Bradshaw.

$\mathrm{Na}$ análise dos pacientes com RCU, a distribuição da doença foi a seguinte: atividade leve em 36 pacientes (63,2\%); atividade moderada $10(17,5 \%)$; e atividade considerada grave em 11 (19,3\%) (Tabela 3 ). Verificou-se correlação significativa, porém fraca, entre o índice de Truelove e Witts e os marcadores lipídicos: HDL-c: $r_{s}=-0,29(p=0,036)$ e CT: $r_{s}=-0,30(p=0,028)$ (Figuras 5 e 6). Não foi observada correlação para os

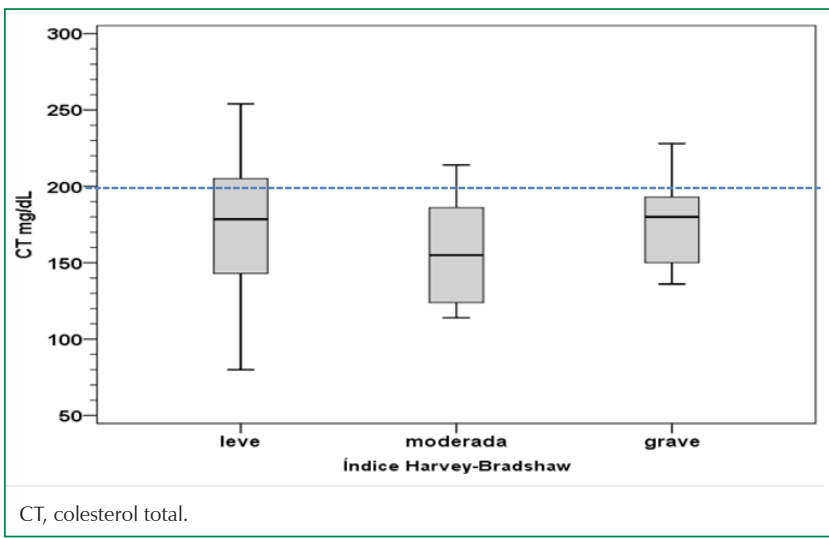

Figura 4. Comparação entre as médias do colesterol total de acordo com a classificação da atividade inflamatória da doença de Crohn pelo Índice de Harvey-Bradshaw.

marcadores LDL-c: $r_{s}=-0,16(p=0,244)$ e TG: $r_{s}=-0,08$ $(\mathrm{p}=0,558)($ Figuras 7 e 8).

O HDL-c de 75 pacientes $(67,6 \%)$ apresentou valores abaixo do recomendado. O LDL-c em 92 pacientes $(83,6 \%)$, o CT em 78 pacientes $(70,9 \%)$ e os TG em 78 pacientes $(70,9 \%)$ da amostra apresentaram valores dentro dos limites recomendados [9].

Tabela 3. Características metabólicas dos pacientes com retocolite ulcerativa conforme a classificação de Truelove e Witts.

\begin{tabular}{lcccc}
\hline \multicolumn{1}{c}{ Variáveis } & $\begin{array}{c}\text { Leve } \\
\text { Média } \pm \text { DP } \\
(\mathrm{n}=36)\end{array}$ & $\begin{array}{c}\text { Moderada } \\
\text { Média } \pm \mathrm{DP} \\
(\mathrm{n}=10)\end{array}$ & $\begin{array}{c}\text { Grave } \\
\text { Média } \pm \mathrm{DP} \\
(\mathrm{n}=11)\end{array}$ & $\mathbf{p}$ \\
\hline PCR $(\mathrm{mg} / \mathrm{dL}$ & $0,89 \pm 0,92$ & $4,1 \pm 10,7$ & $3,0 \pm 2,4$ & 0,114 \\
\hline VHS $(\mathrm{mm})$ & $18,2 \pm 21,8$ & $16,3 \pm 14,8$ & $28,6 \pm 19,8$ & 0,288 \\
\hline HDL-c $(\mathrm{mg} / \mathrm{dL})$ & $60,0 \pm 16,8$ & $52,3 \pm 13,6$ & $48,3 \pm 14,2$ & 0,083 \\
\hline LDL-c $(\mathrm{mg} / \mathrm{dL})$ & $116,0 \pm 41,2$ & $94,7 \pm 18,7$ & $105,6 \pm 26,2$ & 0,236 \\
\hline CT $(\mathrm{mg} / \mathrm{dL})$ & $194,9 \pm 46,5$ & $163,6 \pm 31,0$ & $171,4 \pm 30,0$ & 0,065 \\
\hline TG $(\mathrm{mg} / \mathrm{dL})$ & $112,0 \pm 49,4$ & $123,1 \pm 66,2$ & $98,3 \pm 49,0$ & 0,560 \\
\hline
\end{tabular}

PCR, proteína C reativa; VHS, velocidade de hemossedimentação; HDL-c, colesterol ligado à lipoproteína de alta densidade; LDL-c, colesterol ligado à lipoproteína de baixa densidade; CT, colesterol total; TG: triglicerídeos.

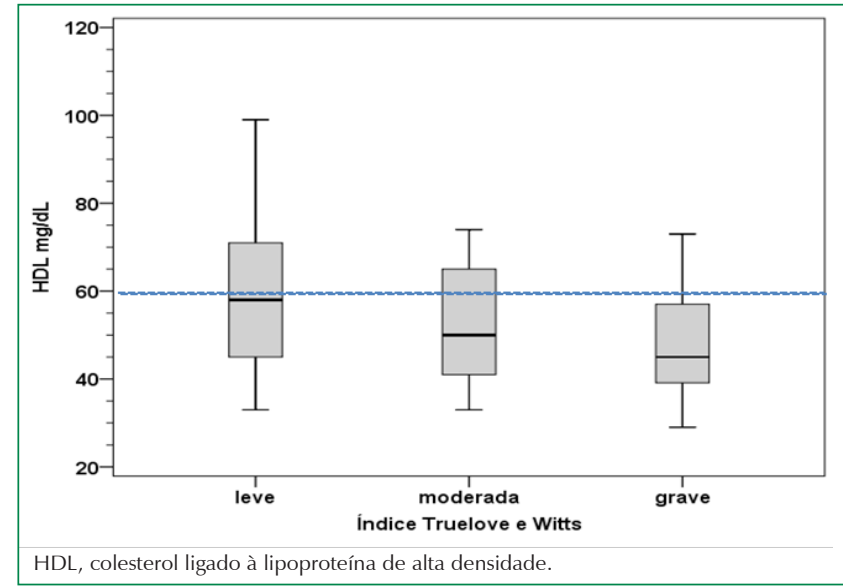

Figura 5. Comparação entre as médias de colesterol ligado à lipoproteína de alta densidade de acordo com a gravidade do surto agudo na retocolite ulcerativa, pelo índice de Truelove e Witts.

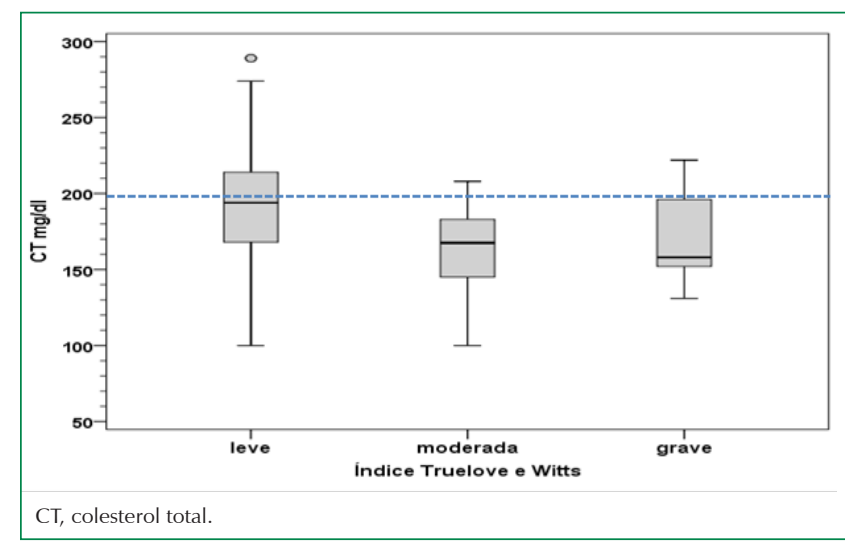

Figura 6. Comparação entre as médias do colesterol total de acordo com a gravidade do surto agudo na retocolite ulcerativa, classificada pelo índice de Truelove e Witts. 


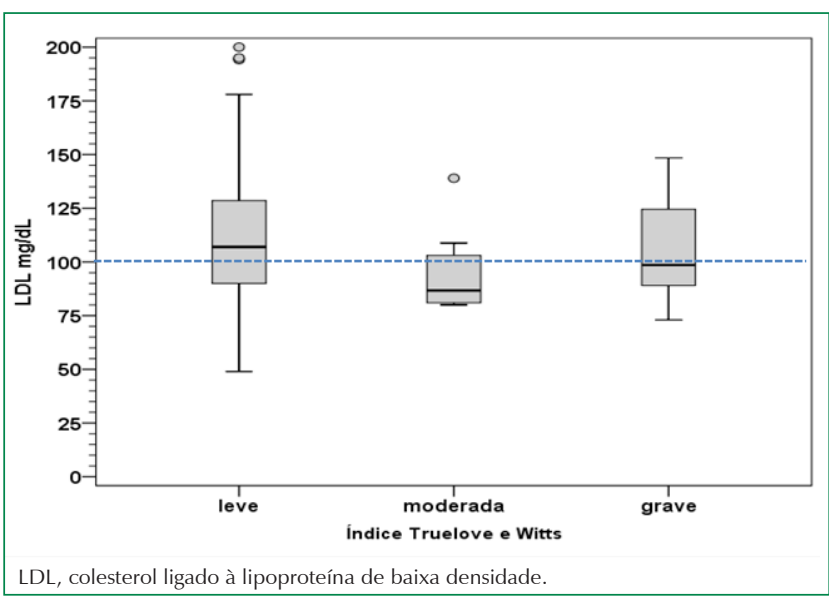

Figura 7. Comparação entre as médias de colesterol ligado à lipoproteína de baixa densidade de acordo com a gravidade do surto agudo na retocolite ulcerativa, classificada pelo índice de Truelove e Witts.

\section{DISCUSSÃO}

No Brasil inexistem estudos epidemiológicos sobre pacientes com DII que envolvam todas as regiões, devido a vários fatores limitantes, entre os quais questões financeiras e logísticas, estruturação de centros especializados e dimensões continentais do país. O presente estudo procurou incluir pacientes com DII de um único centro especializado, em um hospital universitário, para avaliar a situação dessas enfermidades e levantar questões relevantes que possam contribuir para o conhecimento das mesmas.

As DII atingem ambos os sexos e podem ocorrer em qualquer faixa etária, embora predominem em adultos jovens $[2,14]$.Um estudo de 2011 realizado em Joinville com 171 pacientes teve predominância do sexo feminino $(60,8 \%)$ e a média de idade dos pacientes foi de 42,3 anos [15]. O presente estudo encontrou dados semelhantes em relação a média de idade, havendo uma pequena predominância do sexo feminino e da DC em relação à RCU.

Faltam estudos conclusivos em relação ao risco aumentado de aterosclerose em pacientes com DII, considerando-se a possível alteração no perfil lipídico nesse grupo de pacientes. Koutroubakis et al. [16], que acompanharam 22 pacientes com DII antes e após tratamento medicamentoso, encontraram valores médios para HDL-c de 38,6 mg/dL, para LDL-c de $96 \mathrm{mg} / \mathrm{dL}$, para CT de $162,7 \mathrm{mg} / \mathrm{dL}$ e para TG de $115 \mathrm{mg} / \mathrm{dL}$. Uma revisão de 2011, que avaliou os parâmetros do metabolismo lipídico em pacientes com DII, sugere que esses parâmetros podem sofrer alterações em função da produção de citocinas

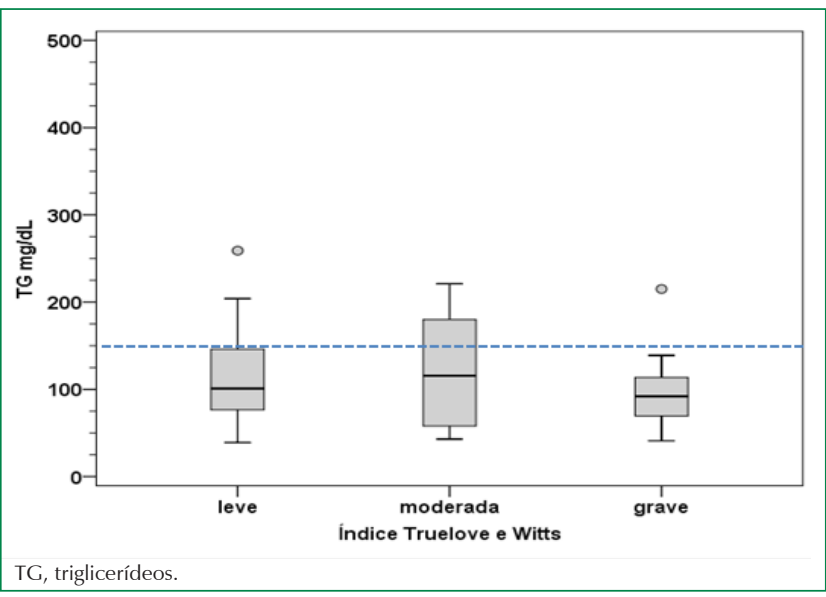

Figura 8. Comparação entre as médias dos triglicerídeos de acordo com a gravidade do surto agudo na retocolite ulcerativa, classificada pelo índice de Truelove e Witts.

envolvidas no processo inflamatório. Os resultados daquela revisão demonstraram níveis baixos de LDL-c e CT, mas não se verificaram alterações significativas nos níveis de HDL-c e TG, que permaneceram estáveis independentemente da gravidade da doença. Mesmo após ressecção intestinal, os níveis de LDL-c permaneciam baixos [17]. Os dados do presente estudo revelam que valores das frações LDL-c, CT e TC mantiveram-se dentro dos limites recomendados pela diretriz da Sociedade Brasileira de Cardiologia [9], enquanto o HDL-c apresentou-se em níveis inferiores aos recomendados.

Romanato et al. [18] não encontraram diferenças significativas no HDL-c, LDL-c e CT em ambas as doenças. Na presente amostra também não se encontraram diferenças significativas em relação às médias de HDL-c, CT e TG entre os grupos, apenas o LDL-c apresentou índice mais elevado na RCU, quando comparado ao grupo de doentes com DC, porém, ainda dentro dos valores recomendados pelas diretrizes, possivelmente não tendo relevância clínica que possa estar relacionada à doença aterosclerótica ou risco cardiovascular.

O IHB é um índice simples e de fácil aplicação, que auxilia na avaliação clínica do paciente com DC, apresentando sensibilidade e especificidade elevadas em relação a outras ferramentas de avaliação [19]. Observou-se que a distribuição da presente amostra em relação à gravidade da doença foi predominantemente de doença leve e moderada, podendo este fato ser explicado por serem pacientes ambula toriais e, consequentemente, mais estáveis e menos graves. 
Por ser uma medida de atividade inflamatória bastante sensível na DC, a PCR é uma importante ferramenta para o acompanhamento da doença e sua atividade. O estudo de Koelewijn et al. [20], que estratificou os pacientes em PCR $\leq 15 \mathrm{mg} / \mathrm{dL}$ e $>15 \mathrm{mg} / \mathrm{dL}$, verificou que níveis mais elevados de PCR estiveram associados a maior gravidade e recaídas da DC. O presente estudo também verificou que a PCR esteve significativamente mais elevada nos pacientes com DC que se apresentavam em estágio grave de inflamação.

Romanato et al. [18] também não encontraram correlação entre os valores de CT e LDL-c com a gravidade da DC. Hrabovský et al. [21] encontraram níveis séricos mais baixos de CT, LDL-c e HDL-c em pacientes com DC em atividade em relação ao grupo controle, porém utilizando critério para avaliar a atividade da doença diferente do que foi utilizado no presente estudo. $\mathrm{Na}$ análise do perfil lipídico dos pacientes com DC da presente amostra em relação à gravidade da doença, também não foram encontrados valores alterados de HDL-c, LDL-c e TG. Não se encontrou correlação entre o IHB e as frações lipídicas analisadas, com exceção do CT que apresentou uma fraca correlação, de pouca relevância clínica.

Para avaliar o grau de atividade da doença na RCU podem-se utilizar os critérios propostos por Truelove e Witts que, por serem de fácil aplicação, podem contribuir para a tomada de decisão quanto ao manejo da doença. Souza et al. [22], utilizando esse índice de avaliação, encontraram doença em estágio leve em $41,4 \%$ da amostra, em pacientes não hospitalizados. Com base nos critérios propostos pelo mesmo índice, o presente estudo encontrou predominância de pacientes com doença leve e moderada. Pode-se considerar que, de forma semelhante aos pacientes com DC, os pacientes com RCU atendidos em ambulatório encontravam-se mais estáveis, em estágio leve de inflamação.

Romanato et al. [18] encontraram valores não significativos para a correlação do LDL-c em relação à atividade da DII. Já o presente estudo identificou correlação significativa entre o índice de Truelove e Witts e as frações lipídicas, apenas para o HDL-c e CT, embora fraca e, possivelmente, de pouca relevância clínica.

Byyani et al. [23] sugerem uma abordagem mais intensa no tratamento de dislipidemia em DII, especialmente quando os níveis de HDL-c forem baixos e o LDL-c aumentado. O HDL-c, por seu efeito anti-inflamatório e por sua participação na redução da síntese de $\mathrm{TNF} \alpha$, pode contribuir para a redução da inflamação na DII [11]. Níveis reduzidos da fração do HDL-c podem estar associados à persistência do quadro inflamatório. Todavia, os dados de Romanato et al. [18] mostraram uma relação inversa entre os níveis de HDL-c e marcadores inflamatórios como PCR e IL-6, tanto na DC como na RCU.

Agouridis et al. [17] não encontraram diferença significativa entre os níveis de HDL-c e TG ou níveis baixos de LDL-c nos pacientes com DII em comparação a indivíduos saudáveis. Em outro estudo, onde 73\% dos pacientes com DII estavam em remissão, foram encontrados níveis de LDL-c e CT baixos tanto na DC como na RCU [24]. No presente estudo, tanto na DC quanto na RCU, verificaram-se níveis adequados de LDL-c, CT e TG, e apenas discreta redução do HDL-c nos pacientes com RCU que se encontravam em grave processo inflamatório, o que pode sugerir menor efeito protetor desse lipídeo na DII.

Não existem estudos para comparação entre os índices IHB e Truelove e Witts e sua correlação com o perfil lipídico dos pacientes com DII. A utilização desse recurso, associada com a caracterização dos marcadores inflamatórios, pode auxiliar na avaliação do paciente e permitir melhor acompanhamento de seu tratamento e evolução da doença. A detecção de alterações nos marcadores inflamatórios e no perfil lipídico pode identificar indivíduos com potencial risco de eventos cardiovasculares, em especial aterotrombóticos, e permitir melhor orientação quanto a aspectos terapêuticos, especialmente quando em presença de dislipidemia. O presente estudo levanta essa questão, reforçando a necessidade de maiores e mais amplos estudos em pacientes com DII, especificamente sobre estes aspectos.

Entre os fatores limitantes, salientamos o tamanho reduzido da amostra e o fato de ter sido recrutada de um único centro de avaliação e tratamento de DII. Recursos limitados de investigação e tempo para realização do estudo também podem ter contribuído. Embora os pacientes não tenham feito uso de estatinas no período do estudo, eles recebiam medicamentos clássicos para o tratamento da DII (corticosteroides, antibióticos, aminossalicilatos, imunossupressores e terapia biológica); porém, não foram feitas análises específicas em relação a essas intervenções terapêuticas. Entendemos, todavia, que essas limitações não interferiram nos resultados, os quais podem contribuir para o melhor entendimento dos pacientes com DII.

Em conclusão, os presentes resultados demonstraram que o LDL-c esteve significativamente mais elevado nos pacientes com RCU quando comparados 
aos pacientes com DC. A fração HDL-c apresentou níveis reduzidos em pacientes com RCU com doença inflamatória mais grave. As demais frações lipídicas não se mostraram alteradas nos dois grupos de pacientes com DII. A PCR aumentada em pacientes com DC esteve associada com a gravidade do processo inflamatório determinada pelo IHB. A detecção dessas alterações metabólicas pode auxiliar na identificação de indivíduos com potencial risco para eventos cardiovasculares.

\section{NOTA}

Declaração de conflitos de interesse

Os autores declaram não haver conflitos de interesse relevantes ao conteúdo deste estudo.

\section{REFERÊNCIAS}

1. Brazilian Study Group of Inflammatory Bowel Diseases. Consensus guidelines for the management of inflammatory bowel disease. Arq Gastroenterol. 2010 Jul-Sep;47(3):313-25. http://dx.doi.org/10.1590/S0004-28032010000300019

2. Freeman HJ. Natural history and long-term clinical course of Crohn`s disease. World J Gastroenterol. 2014 Jan 7;20(1):31-6. http://dx.doi.org/10.3748/wjg.v20.i1.31

3. Van Assche G, Dignass A, Panes J, Beaugerie L, Karagiannis J, Allez M, Ochsenkühn T, Orchard T, Rogler G, Louis E, Kupcinskas L, Mantzaris G, Travis S, Stange E; European Crohn's and Colitis Organisation (ECCO). The second European evidence-based consensus on the diagnosis and management of Crohn`s disease: definitions and diagnosis. J Crohns Colitis. 2010 Feb;4(1):7-27. http://dx.doi.org/10.1016/j.crohns.2009.12.003

4. World Gastroenterology Organisation Practice Guidelines. Doença inflamatória intestinal: uma perspectiva global. Milwaukee, WI: WGO; 2009.

5. Magro F, Cravo M, Lago P, Ministro P, Peixe P, Portela F, Ramos J,Tavares L. Portuguese consensus on the best practice for the management of inflmmatory bowel disease:IBD ahead 2010 meeting results. GE J Port Gastrenterol. 2012 Jul; 19190-8.

6. Harvey R, Bradshaw J. A simpleindex of Crohn's Disease. Lancet. 1980 Mar 8;1(8167):514. http://dx.doi.org/10.1016/S01406736(80)92767-1

7. Dignass A, Eliakim R, Magro F, Maaser C, Chowers Y, Geboes K, Mantzaris G, Reinisch W, Colombel JF, Vermeire S, Travis S, Lindsay JO, Van Assche G. Second European evidence-based consensus on the diagnosis and management of ulcerative colitis Part 1: Definitions and diagnosis. J Crohns Colitis. 2012 Dec;6(10):965-90. http://dx.doi.org/10.1016/j. crohns.2012.09.003

8. Hanauer SB, Rubin DT, Sandborn WJ. Treatment guidelines and clinical practice: optimizing foundational therapies for ulcerative colitis. Gastroenterol Hepatol. 2008 Oct;4(10 Suppl 22):1-16.

9. Sociedade Brasileira de Cardiologia. V Diretriz brasileira sobre dislipidemias e prevenção da aterosclerose. Arq Bras Cardiol. 2013;101(4 Supl 1):1-22. http://dx.doi.org/10.5935/abc.2013S010

10. Gandhi S, Narula N, Marshall JK, Farkouh M. Are patients with inflammatory bowel disease at increased risk of coronary artery disease? Am J Med. 2012 Oct;125(10):956-62. http://dx.doi.org/10.1016/j.amjmed.2012.03.015

11. Williams HR, Willsmore JD, Cox IJ, Walker DG, Cobbold JF, Taylor-Robinson SD, Orchard TR. Serum metabolic profiling in inflammatory bowel disease. Dig Dis Sci. 2012 Aug;57(8):2157-65. http://dx.doi.org/10.1007/s10620-012-2127-2

12. Andersen NN, Jess T. Risk of cardiovascular disease in inflammatory bowel disease. World J Gastrointest Pathophysiol. 2014 Aug 15;5(3):359-65.

13. Cardozo WS, Sobrado CW. Doença inflamatória intestinal. 2a ed. Barueri, SP: Editora Manole; 2015.

14. Elia PP, Fogaça HS, Barros RGGR, Zaltman C, Elia CSC. Análise descritiva dos perfis social,clínico,laboratorial e antropométrico de pacientes com doenças inflamatórias intestinais, internados no Hospital Universitários Clementino Fraga Filho, Rio de Janeiro. Arq Gastroenterol. 2007;44(4):332-9. http://dx.doi.org/10.1590/S0004-28032007000400010

15. Victoria CR, Sassaki LY, Nunes HRC. Incidence and prevalence rates of inflammatory bowel diseases, in midwestern of São Paulo state, Brazil. Arq Gastroenterol. 2009;46(1):20-4. http://dx.doi.org/10.1590/S0004-28032009000100009

16. Koutroubakis IE, Oustamanolakis P, Malliaraki N, Karmiris K, Chalkiadakis I, Ganotakis E, Karkavitsas N, Kouroumalis EA. Effects of tumor necrosis factor alpha inhibition with infliximab on lipid levels and insulin resistance in patients with inflammatory bowel disease. Eur J Gastroenterol Hepatol. 2009 Mar;21(3):283-8. http://dx.doi.org/10.1097/ MEG.0b013e328325d42b

17. Agouridis AP, Elisaf M, Milionis HJ. An overview of lipid abnormalities in patients with inflammatory bowel disease. Ann Gastroenterol. 2011;24(3):181-7.

18. Romanato G1, Scarpa M, Angriman I, Faggian D, Ruffolo C, Marin R, Zambon S, Basato S, Zanoni S, Filosa T, Pilon F, Manzato E. Plasma lipids and inflammation in active inflammatory bowel diseases. Aliment Pharmacol Ther. 2009 Feb 1;29(3):298-307. 
19. Vermeire S, Schreiber S, Sandborn WJ, Dubois C, Rutgeerts P. Correlation between the cronh`s disease activity and harveybradshaw indices is assessing crohn`s disease severity. Clin Gastroenterol Hepatol. 2010 Apr;8(4):357-63. http://dx.doi. org/10.1016/j.cgh.2010.01.001

20. Koelewijn CL, Schwartz MP, Samsom M, Oldenburg B. C-reactive protein levels during a relapse of Crohn's disease are associated with the clinical course of the disease. World J Gastroenterol. 2008 Jan 7;14(1):85-9. http://dx.doi.org/10.3748/ wjg. 14.85

21. Hrabovský V1, Zadák Z, Bláha V, Hyspler R, Karlík T, Martínek A, Mendlová A. Cholesterol metabolism in active Crohn's disease. Wien Klin Wochenschr. 2009;121(7-8):270-5. http://dx.doi.org/10.1007/s00508-009-1150-6

22. Souza MM, Belasco AGS, Aguilar-Nascimento JE. Perfil epidemiológico dos pacientes portadores de doença inflamatória intestinal do estado de Mato Grosso. Rev Bras Coloproct. 2008;28(3):324-8. http://dx.doi.org/10.1590/S0101-98802008000300009

23. Sappati Biyyani RS, Putka BS, Mullen KD. Dyslipidemia and lipoprotein profiles in patients with inflammatory bowel disease. J Clin Lipidol. 2010 Nov-Dec;4(6):478-82. http://dx.doi.org/10.1016/j.jacl.2010.08.021

24. Theocharidou E, Tellis CC, Mavroudi M, Soufleris K, Gossios TD, Giouleme O, Athyros VG, Tselepis AD, Karagiannis A. Lipoprotein-associated phospholipase A2 and arterial stiffness evaluation in patients with inflammatory bowel diseases. J Crohns Colitis. 2014 Sep;8(9):936-44. http://dx.doi.org/10.1016/j.crohns.2014.01.016 